\title{
Bone marrow stromal cells promote neuromotor functional recovery, via upregulation of neurotrophic factors and synapse proteins following traumatic brain injury in rats
}

\author{
YAN FENG $^{1}$, YARU JU ${ }^{2}$, JIANZHONG CUI ${ }^{3}$ and LIQUN WANG ${ }^{1}$ \\ ${ }^{1}$ Department of Neurosurgery, The Second Hospital of Hebei Medical University, Shijiazhuang, \\ Hebei 050000; ${ }^{2}$ Department of Obstetrics, The Fourth Hospital of Shijiazhuang, Shijiazhuang, Hebei 050011; \\ ${ }^{3}$ Department of Neurosurgery, Tangshan Workers' Hospital, Tangshan, Hebei 063000, P.R. China
}

Received January 23, 2016; Accepted February 6, 2017

DOI: $10.3892 / \mathrm{mmr} .2017 .6619$

\begin{abstract}
It has previously been demonstrated that bone marrow stromal cells (BMSCs) exhibit great therapeutic potential in neuronal injuries; however, there is limited understanding of the precise underlying mechanisms that contribute to functional improvement following brain injury. The aim of the present study was to assess the effect of BMSC treatment on traumatic brain injury (TBI) in rats, and investigate if they migrate to injured areas and promote neuromotor functional recovery via upregulation of neurotrophic factors and synaptic proteins. BMSCs were cultured in vitro from female Sprague Dawley (SD) rat bone marrow and were subsequently infused into male adult SD rats via the tail vein, following induction of TBI. The results demonstrated that treatment with BMSCs significantly reduced TBI-induced neuromotor impairment and neuronal loss, as assessed by rota rod testing, western blot analysis, modified neurological severity score and immunohistochemistry. The distribution of transplanted BMSCs was tracked by monitoring the expression of sex determining region $\mathrm{Y}$ (SRY) in rats. The number of cells double-positive for SRY/neuronal nuclear antigen or SRY/glial fibrillary acidic protein was increased in the BMSC group, which demonstrated that BMSCs migrated to injured areas and differentiated into neurons and astrocytes, following TBI.
\end{abstract}

Correspondence to: Dr Liqun Wang, Department of Neurosurgery, The Second Hospital of Hebei Medical University, 215 Heping West Road, Shijiazhuang, Hebei 050000, P.R. China

E-mail: lqwangdr@163.com

Abbreviations: BMSCs, bone marrow stromal cells; mNSS, modified neurologic severity score; VEGF, vascular endothelial growth factor; BDNF, brain derived neurotrophic factor; SRY, sex determining region Y; SYN, synaptophysin; DAPI, 4',6-diamidino-2-phenylindole

Key words: bone marrow stromal cells, traumatic brain injury, vascular endothelial growth factor, brain derived neurotrophic factor, sex determining region $\mathrm{Y}$, synaptophysin
Furthermore, administration of BMSCs increased expression of vascular endothelial growth factor and brain derived neurotrophic factor. Protein expression levels of synaptophysin were downregulated following TBI and this was reversed in part by treatment with BMSCs. These findings uncovered some underlying mechanisms of action of BMSCs, and may lead to their potential use as a future effective therapeutic agent for the treatment of TBI.

\section{Introduction}

Traumatic brain injury (TBI) is a primary health concern and leading cause of mortality worldwide. Neurological deficits resulting from TBI present a severe burden to patient families and society. There is no current effective treatment method to promote functional recovery except for routine rehabilitation, hyperbaric oxygen and basic care. It has previously been demonstrated that neural stem cells (NSCs) may promote neurological recovery following brain injury (1). Stem cell therapy is a cellular approach that has the potential to induce a variety of beneficial neurorestorative processes that may facilitate the recovery of neurological function (2). Over the last decade, bone marrow stromal cells (BMSCs) have been used as therapeutic vectors or tools for the treatment of a variety of diseases. Previous studies have demonstrated that the transplantation of BMSCs in animal models of injury exhibits protective effects following acute spinal cord (3), lung (4) and liver (5) injuries. BMSCs have been demonstrated improve the neurological functional outcome of central nervous system (CNS) disorders, including stroke and TBI $(6,7)$. Furthermore, BMSCs are capable of proliferating and differentiating into neurons, astrocytes or oligodendrocytes in vitro $(8,9)$.

Previous studies have revealed a significant loss of synapses in the days following brain injury, notably in the brain regions connected to the site of initial injury, including the hippocampus (10). Post-traumatic neuronal plasticity involves aspects of neurogenesis, including axonal sprouting, synaptic formation and remodeling. Previous studies have reported that BMSCs increase synapse protein expression in the ischemic brain and reconstructed neuronal networks $(11,12)$. A further study reported that statin therapy had a synergetic effect with 
BMSCs, and mobilized engrafted BMSCs to the lesion area, promoting repair following stroke (13). The transplantation of BMSCs is therefore considered a promising strategy for TBI treatment and may promote morphological and functional recovery post-TBI. However, the neuroprotective effect and underlying mechanisms of BMSCs following TBI require further examination and remain to be fully elucidated.

The present study hypothesized that the effect of BMSCs on motor function may be associated with the expression of synaptophysin (SYN). Therefore, a rat model of TBI was constructed to investigate if BMSCs migrate to injured areas and promote functional recovery via upregulation of neurotrophic factors and synaptic proteins.

\section{Materials and methods}

Cell culture. A total of 15 Sprague-Dawley (SD) female rats (age, 1 month; weight, 20-24 g), were obtained from the Hebei Medical University Experimental Animal Center (Shi Jiazhuang, China) and were housed in a temperature-controlled $\left(22-24^{\circ} \mathrm{C}\right)$ room with a 12 -h light/dark cycle and with water and food freely available. Rats were anaesthetized with $10 \%$ chloral hydrate $(3 \mathrm{ml} / \mathrm{kg}$; Bio-Rad Biotechnology, Inc., Shanghai, China) and BMSCs were isolated and cultured. Briefly, fresh bone marrow cells were collected from the femurs of SD rats, via suction from the medullary cavity using a $20-\mathrm{ml}$ sterile syringe. All rats were sacrificed following cell harvesting. For anticoagulation, $5 \mathrm{ml}$ heparin $(100 \mathrm{IU} / \mathrm{ml})$ was used. Following filtration, cells were centrifuged at $1,000 \mathrm{x} \mathrm{g}$ for $5 \mathrm{~min}$ at $4^{\circ} \mathrm{C}$. The purified cells were cultured in a $25 \mathrm{~cm}^{2}$ flask with Dulbecco's modified Eagle's medium/nutrient mixture F12 (DMEM/F12; Gibco; Thermo Fisher Scientific, Inc., Waltham, MA, USA) supplemented with $10 \%$ fetal bovine serum (Hyclone; GE Healthcare, Logan, UT, USA), 100 U/ml penicillin and $100 \mu \mathrm{g} / \mathrm{ml}$ streptomycin (Sigma-Aldrich; Merck Millipore, Darmstadt, Germany), and incubated at $37^{\circ} \mathrm{C}$ in $5 \%$ $\mathrm{CO}_{2}$. After $48 \mathrm{~h}$, non-adherent cells were removed and fresh media was added. When adherent cells reached $\sim 80 \%$ confluency, the cells were dissociated with $0.25 \%$ trypsin solution and re-seeded again. Following three passages of culture, passage 3 BMSCs were used for subsequent experiments.

Animals and TBI model. Adult male SD rats (weight, 250-300 g; age, 12-16 weeks), were obtained from Hebei Medical University Experimental Animal Center and were housed in a temperature-controlled $\left(22-24^{\circ} \mathrm{C}\right)$ room with a 12-h light/dark cycle and with water and food freely available. A total of 105 rats were utilized in this study. All experimental procedures were performed in accordance with the guidelines of the Chinese council on animal protection, and were approved by the Hebei Medical University Committee (Shijiazhuang, China) for the use of animals in research (Permit Number: 2015046). The TBI model was developed as described using a weight-drop device (14). Briefly, the rats were anaesthetized with $10 \%$ chloral hydrate $(3 \mathrm{ml} / \mathrm{kg})$ and fixed onto the stereotactic device. Aseptic techniques were used throughout surgery. A midline incision was made to expose the skull and right parietal craniotomy $(5-\mathrm{mm}$ in diameter, $1.5 \mathrm{~mm}$ posterior, and $2.5 \mathrm{~mm}$ lateral to the bregma) using a high-speed microdrill. The dura was exposed and kept intact.
A 40-g steel weight fell freely through a vertical tube from $2.5 \mathrm{~m}$ onto the motor cortex to induce TBI. Following injury, the bone flap was placed in situ and the scalp was sutured. Rats were placed on heat pads $\left(37^{\circ} \mathrm{C}\right)$ for $4 \mathrm{~h}$ to maintain normal body temperature during the recovery period. Sham-operated animals underwent procedures identical to those of the TBI animals, including anesthesia and surgery; however, did not receive TBI.

Groups and drug administration. The 105 adult rats were randomly divided into 3 groups ( $n=35 /$ group): Sham, TBI and TBI + BMSC-treated. Prior to transplantation, BMSCs were digested with trypsin, washed twice with DMEM and centrifuged at $1,000 \mathrm{x}$ g for $5 \mathrm{~min}$ at $4^{\circ} \mathrm{C}$. In BMSC-transplanted animals, the BMSCs $\left(3 \times 10^{6}\right.$ cells $\left./ \mathrm{ml}\right)$ in $1 \mathrm{ml}$ of phosphate-buffered saline (PBS) were transplanted via a tail vein puncture into rats $30 \mathrm{~min}$ following the induction of TBI. The sham and TBI groups received equal volumes of saline injection. Each subgroup was composed of five rats, and rats were anesthetized with $10 \%$ chloral hydrate and decapitated 1 , 3, 5, 7 and 14 days following TBI. The remainder of the rats ( $n=10$ per treatment group) underwent neurobehavioral examinations. All investigations were blind and the groups were revealed at the end of the behavioral and histological analyses.

Rotarod task. A rotarod was used to assess motor function as previously described (15). Briefly, a 7-cm diameter cylinder was positioned $1.2 \mathrm{~m}$ above a foam pad while speed and acceleration were controlled by computer interface (San Diego Instruments, San Diego, CA, USA). For each trial, the rat was placed on the rotating barrel, the speed was accelerated from 4 to $40 \mathrm{rpm}$ over a period of $5 \mathrm{~min}$, and the latency to fall was recorded. Pre-training occurred once a day for the 3 days preceding injury. Following injury, rats were re-tested on the rotarod task on days 1, 3, 5, 7 and 14 post-TBI. Each rat underwent four test trials per day. The average latency of the total of the four trials was calculated.

Modified neurological severity score ( $m N S S)$. Neurological deficits were evaluated using the mNSS on an 18-point scale by a researcher blinded to treatment, which tested reflexes, alertness, coordination and motor abilities. One point was awarded for failure to perform a particular task; thus, the higher the score, the more severe the injury, whereas a healthy rat scored zero. Post-injury, mNSS was evaluated at days 1-14 post-TBI.

Histological analysis. Brain tissues were fixed in $4 \%$ paraformaldehyde solution for $24 \mathrm{~h}$, washed with running water for $4 \mathrm{~h}$, and embedded in paraffin and dehydrated with gradient alcohol and xylene. The samples were serially sectioned at a thickness of $5 \mu \mathrm{m}$. All sections were mounted on glass slides and subsequently stained with hematoxylin and eosin (H\&E). Sections were observed and analyzed using an optical microscope.

Western blot analysis. Rats were decapitated under deep anesthesia and the brains were rapidly isolated. The hippocampal tissues were dissected on ice, the proteins were extracted using radioimmunoprecipitation assay buffer (Beyotime Institute of Biotechnology, Shanghai, China) from the cortex surrounding the injured area and the protein concentration was determined 
using a bicinchoninic acid kit (Beijing Solarbio Science \& Technology Co., Ltd., Beijing, China). Samples (50 $\mu \mathrm{g}$ ) were separated by $10 \%$ SDS-PAGE and subsequently transferred onto polyvinylidene membranes (Roche Diagnostics $\mathrm{GmbH}$, Mannheim, Germany). The blots were blocked with 5\% fat-free dry milk for $2 \mathrm{~h}$ at room temperature, followed by incubation with the following rabbit primary antibodies: Anti-vascular endothelial growth factor (VEGF) polyclonal (1:1,000; Abcam, ab53465, Cambridge, UK), anti-brain derived neurotrophic factor (BDNF) monoclonal (1:1,000; Abcam, ab216443) and anti- $\beta$-actin monoclonal (1:1,000; Affinity Biologicals Inc., AF7018, Ancaster, ON, Canada) at $4^{\circ} \mathrm{C}$ overnight. Following this, the membranes were incubated with horse-radish peroxidase (HRP)-conjugated donkey anti-mouse immunoglobulin (Ig)-G or donkey anti-rabbit IgG (sc-2314 and sc-2313; 1:5,000; both from Santa Cruz Biotechnology, Inc., Dallas, TX, USA) secondary antibodies at $37^{\circ} \mathrm{C}$ for $1 \mathrm{~h}$. Signals were detected by Enhanced Chemiluminescence using a Western Lightning ${ }^{\circledR}$ Plus-ECL kit (Perkin-Elmer, Inc., Waltham, MA, USA). (Densitometric analysis for the blots was performed using National Institutes of Health Image software version 1.41 (Bethesda, MD, USA).

Immunohistochemical and immunofluorescence analyses. Rats were perfused transcardially with saline under deep anesthesia, followed by $4 \%$ paraformaldehyde for $24 \mathrm{~h}$, and placed into $30 \%$ sucrose solution (0.1 M PBS, pH 7.4) until they sank to the bottom. The brain tissues were embedded in optimum cutting temperature compound and cut into 15 $\mu \mathrm{m}$-thick sections coronally from the anterior to posterior hippocampus (bregma -2.0 to $-3.0 \mathrm{~mm}$ ) using a cryostat. Frozen sections were sliced with a microtome, treated with $0.4 \%$ Triton X-100 for 20 min and blocked at room temperature in normal donkey serum (017-000-121; Shanghai Solarbio Bioscience \& Technology Co., Ltd., Shanghai, China) for $2 \mathrm{~h}$. For immunohistochemical analyses, sections were incubated overnight at $4^{\circ} \mathrm{C}$ with rabbit anti-VEGF (1:100) and rabbit anti-BDNF polyclonal antibodies (1:100), and subsequently with HRP-conjugated anti-rabbit IgG antibodies at $37^{\circ} \mathrm{C}$ in the dark for $30 \mathrm{~min} .3,3^{\prime}$-Diaminobenzidine was used to reveal the immunohistochemical reaction. For double labeling, the frozen sections were incubated with rabbit anti-sex determining region Y (SRY) polyclonal (1:100; Abcam, ab209858), mouse anti-neuronal nuclear antigen (NeuN) monoclonal (1:100; EMD Millipore, Billerica, MA, USA, MAB324-K) or anti-glial fibrillary acidic protein (GFAP) monoclonal (1:100; EMD Millipore, IF03L) antibodies overnight at $4^{\circ} \mathrm{C}$. The following day, the sections were incubated with fluorescein isothiocyanate-conjugated anti-rabbit IgG or anti-mouse $\operatorname{IgG}$ secondary antibodies (sc-2090 and sc-2099; 1:1,000; Santa Cruz Biotechnology, Inc.) for $2 \mathrm{~h}$ at $37^{\circ} \mathrm{C}$ in the dark. All cell nuclei were counterstained with 4',6-diamidino-2-phenylindole (DAPI). PBS was substituted for the primary antibody as the negative control. Sections were imaged under a laser scanning confocal microscope (Olympus Fluoview ${ }^{\mathrm{TM}}$ FV1000; Olympus Corporation, Tokyo, Japan).

Statistical analysis. All experiments were repeated three times and similar results were obtained. Statistical analysis was performed using SPSS software, version 16.0 (SPSS, Inc.,

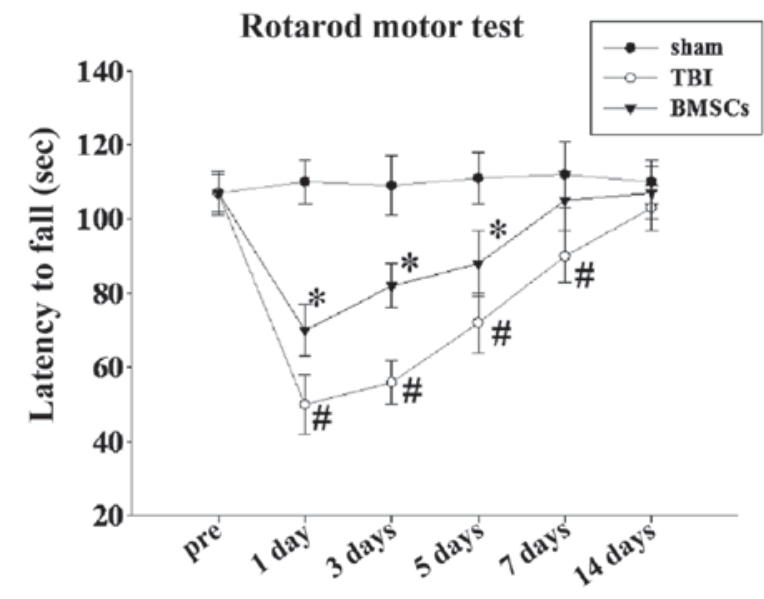

Figure 1. BMSC treatment improves motor deficits. Motor dysfunction was assessed at 1, 3, 5, 7 and 14 days following induction of TBI. The TBI group rat had shorter times than the sham group rats on days $1,3,5$ and 7 post-trauma. TBI rats regained the function of the sham animals by day 14 The BMSC group rats had longer times than TBI rats on days 1,3 , and 5 post-trauma. Data are expressed as the mean \pm standard deviation. ${ }^{*} \mathrm{P}<0.01$ vs. sham group; ${ }^{~} \mathrm{P}<0.05$ vs. TBI group. TBI, traumatic brain injury; BMSCs, bone marrow stromal cells.

Chicago, IL, USA). Data are expressed as the mean \pm standard deviation and the significance of the experimental results was determined using one-way analysis of variance followed by the Student-Newman-Keuls post hoc multiple comparisons test. $\mathrm{P}<0.05$ was considered to indicate a statistically significant difference.

\section{Results}

BMSC treatment improves motor deficits. To determine the neuroprotective effects of BMSCs against TBI-induced brain damage, the present study examined the effects of BMSCs on motor deficits via a rotarod task and mNSS score following TBI at 1, 3, 5, 7 and 14 days. As presented in Fig. 1, TBI resulted in a significant motor deficit at 1, 3, 5 and 7 days compared with the sham group, and BMSC treatment significantly improved motor function recovery and latency(s) at 1, 3 and 5 days compared with the TBI group. In addition, it was demonstrated that the mNSS of the rats in the TBI group was significantly increased in comparison with the sham group at 1-7 days, and BMSC treatment significantly reduced the mNSS score compared with the TBI group at 1-5 days (Fig. 2), and additionally improved neuromotor function.

BMSC treatment reduces cortex neuronal death. H\&E staining was performed to examine the effect of BMSCs on ipsilateral cerebral cortex neuronal damage 7 days following TBI. In the TBI rats, there were marked morphological alterations in the cortex compared with the sham group. Neuronal cell body swelling and disorder was observed, in addition to intercellular broadening, cell loss and nuclear pyknosis and karyolysis in the TBI group (Fig. 3A). Treatment with BMSCs significantly moderated morphological alterations and reduced neuronal loss induced by injury (Fig. 3B, ${ }^{*} \mathrm{P}<0.01$ vs. sham group; ${ }^{\text {}} \mathrm{P}<0.05$ vs. TBI group). 


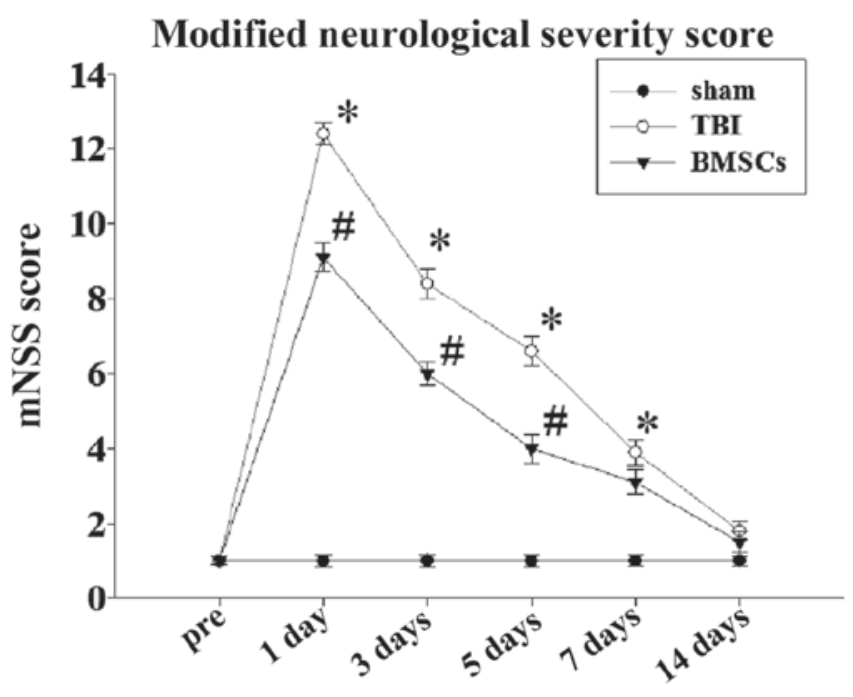

Figure 2. Effect of BMSCs on mNSS score. The mNSS of rats in the TBI group were significantly increased in comparison with the sham group at $1,3,5$ and 7 days, and administration of BMSCs significantly improved neuromotor function at 1,3 and 5 days following TBI, as reflected by a decrease in mNSS. Data are expressed as the mean \pm standard deviation. ${ }^{\text {}} \mathrm{P}<0.01$ vs. sham group; ${ }^{~} \mathrm{P}<0.05$ vs. TBI group. TBI, traumatic brain injury; BMSCs, bone marrow stromal cells; mNSS, modified neurological severity score.

A
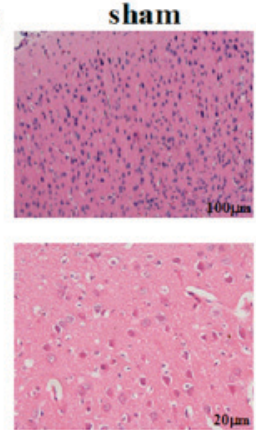

B

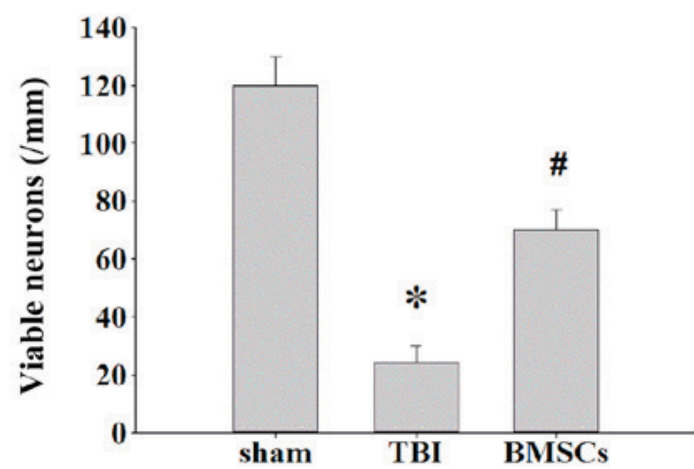

Figure 3. Effect of BMSCs on cortex neuronal damage assessed via hematoxylin and eosin staining. (A) Representative staining in ipsilateral cerebral cortex areas of sham, TBI and BMSCs treatment groups at 7 days following TBI. Scale bar, 100 and $20 \mu \mathrm{m}$. (B) Quantification of the number of viable neurons/mm hippocampal area in each group. Data are expressed as the mean \pm standard deviation ( $\mathrm{n}=5 /$ per group). ${ }^{*} \mathrm{P}<0.01$ vs. sham group; ${ }^{*} \mathrm{P}<0.05$ vs. TBI group. TBI, traumatic brain injury; BMSCs, bone marrow stromal cells.

$B M S C$ treatment increases expression of $V E G F$ and $B D N F$. The expression of VEGF and BDNF was detected at 14 days following TBI via immunochemistry and western blot assay.
A

TBI

TBI

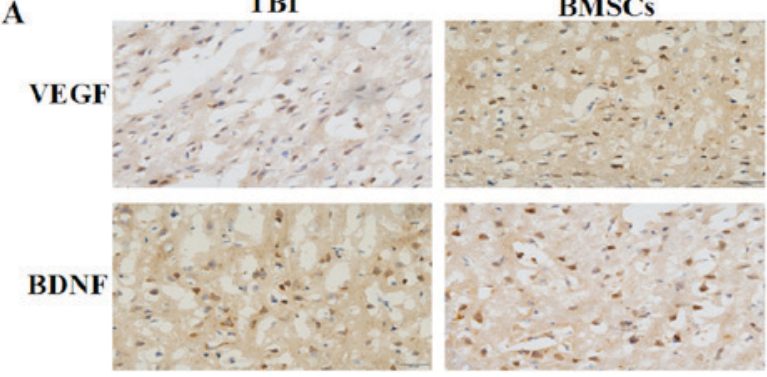

B

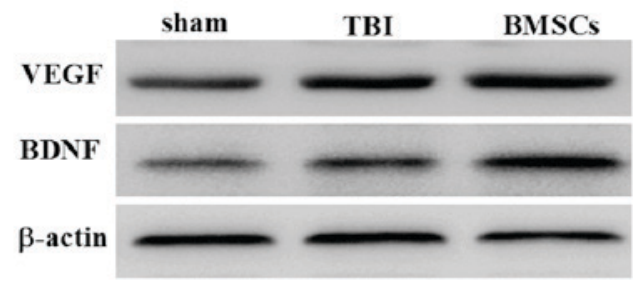

$\mathrm{C}$
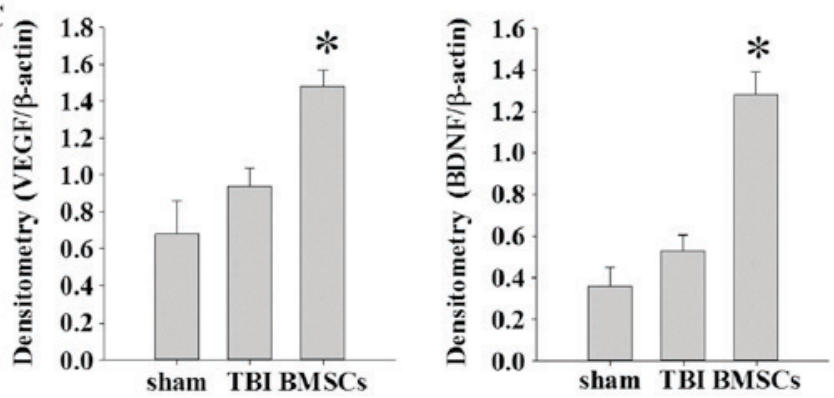

Figure 4. Effect of BMSCs on the expression of VEGF and BDNF in the ipsilateral cerebral cortex. (A) The expression of VEGF and BDNF was determined via immunohistochemical staining. Scale bar, $50 \mu \mathrm{m}$. (B) Western blot images representing protein expression levels of VEGF and BDNF in the ipsilateral cerebral cortex of rats at 14 days. (C) Densitometry analysis of VEGF and BDNF bands corresponding to $\beta$-actin. ${ }^{*} \mathrm{P}<0.05$ vs. TBI group. TBI, traumatic brain injury; BMSCs, bone marrow stromal cells; VEGF, vascular endothelial growth factor; BDNF, brain derived neurotrophic factor.

As presented in Fig. 4A, weak immunopositive staining was observed in the sham group; however, strong immunohistochemical staining for VEGF or BDNF was observed in the cytoplasm of cells around the injury site in the BMSC group. The protein expression levels of VEGF and BDNF were significantly increased in the BMSC group compared with the sham and TBI groups (Fig. 4B and $\mathrm{C} ;{ }^{*} \mathrm{P}<0.05$ ).

BMSCs may migrate to injured areas and differentiate into neurons or astrocytes. BMSCs were tracked to evaluate their migration and distribution patterns in rats. BMSCs isolated from female rats were injected into male rats in vivo. The expression of SRY was detected via laser scanning confocal microscopy to trace transplanted BMSCs in the injured site. Double immunofluorescence staining was performed to investigate the co-localization of SRY and NeuN or GFAP expression. As presented in Fig. 5, SRY was stained with rabbit anti-SRY antibody and secondary antibodies labeled with red fluorescence. In addition, neurons or astrocytes were stained with mouse anti-NeuN antibody or mouse anti-GFAP antibody and secondary antibody labeled with green fluorescence. Sections were stained with DAPI (blue) to reveal all nuclei. The images were merged, and various SRY-positive cells in the lesion epicenter were observed to be positive for $\mathrm{NeuN}$ 
A

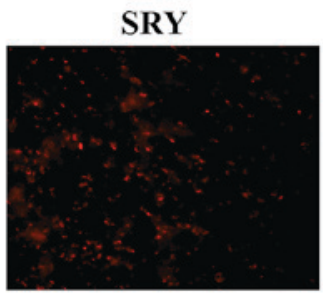

SRY

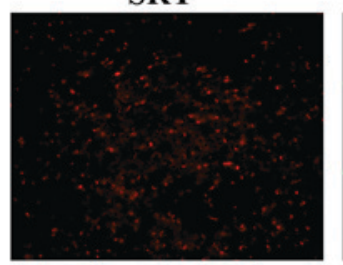

B

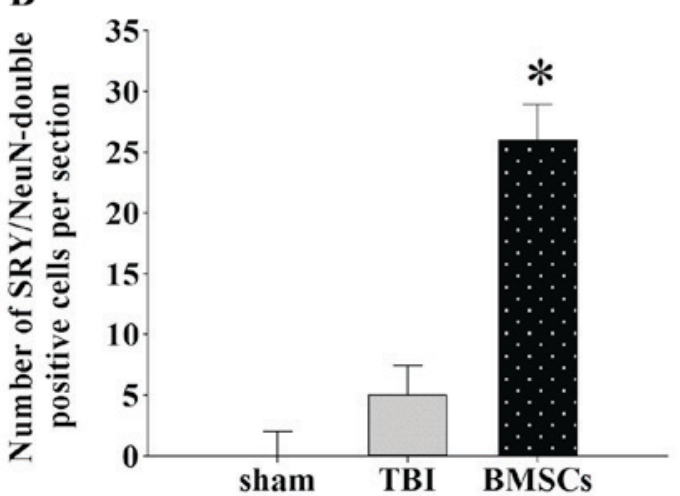

DAPI

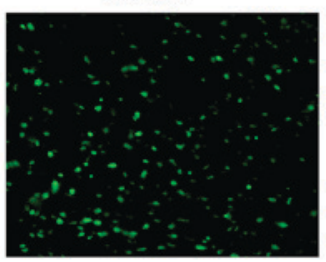

GFAP
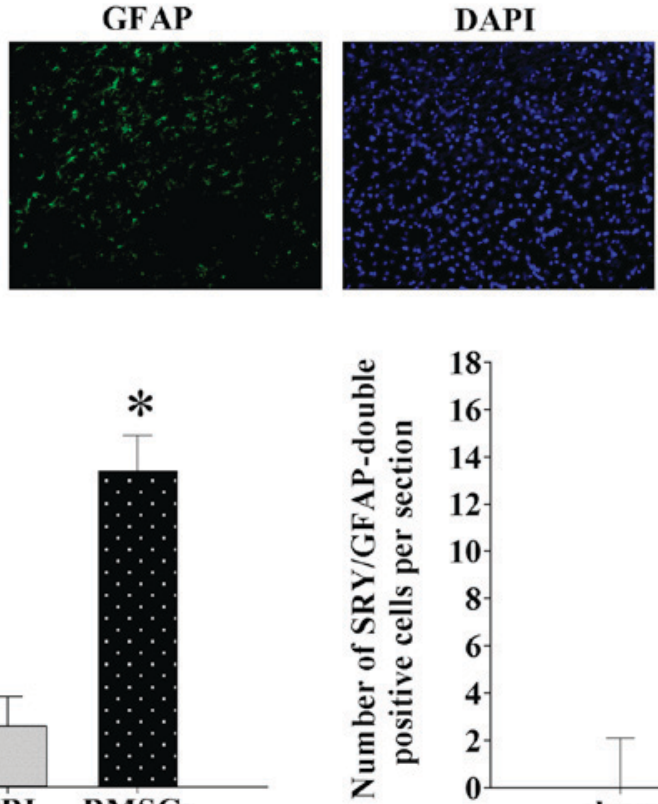
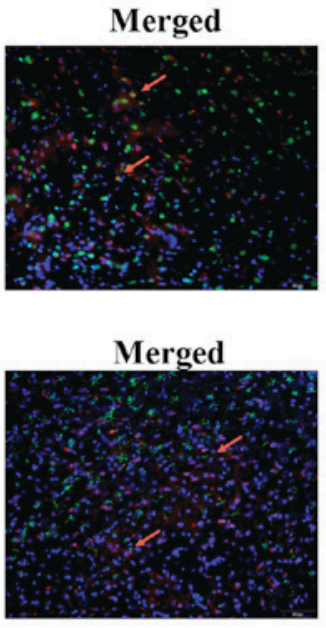

187

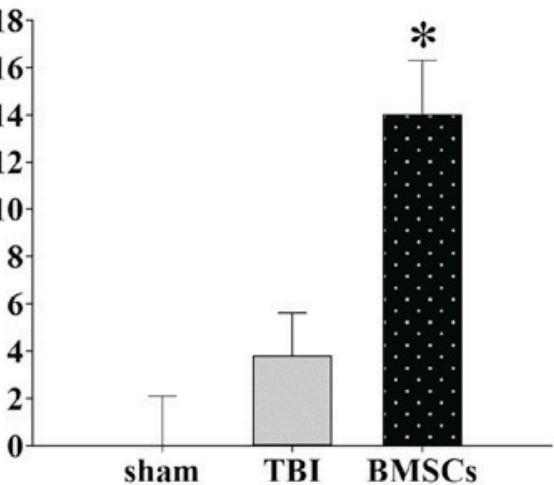

Figure 5. BMSCs may migrate to injured areas and differentiate into neurons or astrocytes. (A) Representative images of co-localization of SRY and NeuN or GFAP at 14 days following TBI, as determined via immunofluorescent staining and counterstaining cell nuclei with DAPI. Orange labeling in images indicates co-localization. Scale bar $=50 \mu \mathrm{m}$. (B) Quantification of results demonstrating that the BMSC-treated group exhibited significantly greater NeuN or GFAP-positive cells co-labeled with SRY in the ipsilateral cortex compared with the TBI group. Data are expressed as the mean per field of view (n=5/group). ${ }^{*} \mathrm{P}<0.01$ vs. TBI group. TBI, traumatic brain injury; BMSCs, bone marrow stromal cells; SRY, sex determining region Y; NeuN, neuronal nuclear antigen; GFAP, glial fibrillary acidic protein.

or GFAP at 14 days following TBI (Fig. 5A). The number of cells double-positive for SRY/NeuN or SRY/GFAP was greater in the BMSC group compared with the TBI group (Fig. 5B; $\left.{ }^{*} \mathrm{P}<0.01\right)$. These results suggested that BMSCs migrate to injured areas and differentiate into neurons and astrocytes following induction of TBI in the rat.

BMSC treatment attenuates synapse protein loss. To further investigate the BMSC underlying mechanisms of action, western blotting was performed to examine the expression of SYN at 1, 3, 5, 7 and 14 days post-TBI (Fig. 6A). As presented in Fig. 6 and B, there was a significant downregulation of SYN expression in the TBI group compared with the sham group at 1,3 and 5 days $\left({ }^{*} \mathrm{P}<0.05\right)$. Reduced levels of SYN in TBI animals indicates loss of synapses. Treatment with BMSCs resulted in significantly greater levels of SYN at 1-5 days compared with the TBI group (Fig. 6; ${ }^{*} \mathrm{P}<0.05$ ).

\section{Discussion}

The results from the present study are similar to the findings of previous investigations, indicating that in animal models of TBI or stroke, BMSCs may effectively reduce brain damage and improve functional recovery $(6,13,16)$. The present study verified and expanded previous results by demonstrating that BMSCs migrate to injured brain tissue, reduce motor deficits and neuronal injuries, increase the expression of VEGF and BDNF and induce a greater expression of synaptophysin following TBI. These results thus demonstrated that BMSCs exhibit potential as an effective treatment to promote recovery following TBI.

TBI is a highly complex disorder, resulting from injury to primary and secondary brain signaling pathways. Currently, there is no effective treatment for brain injury to promote functional recovery, except for routine rehabilitation and basic care. Notably, BMSCs have previously been demonstrated to improve neurological functional recovery in experimental TBI models. There are various explanations regarding the broad underlying mechanisms by which BMSCs exert their beneficial effects. Previous findings indicated that injected BMSCs cross the blood brain barrier and actively migrate to sites of tissue damage $(17,18)$. Additionally, BMSCs expressing C-X-C chemokine receptor type 4 have an active tropism toward zones of tissue damage where the expression of stromal cell derived factor (SDF)-1 is increased (19). The tracking of BMSCs is essential for evaluation of their migration and distribution patterns in rats following TBI. The present study harvested BMSCs from female rats, cultured them and injected them into 
A

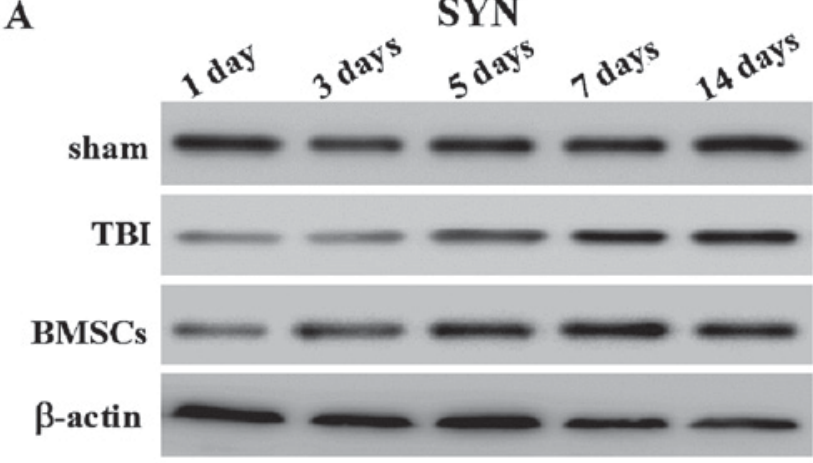

B

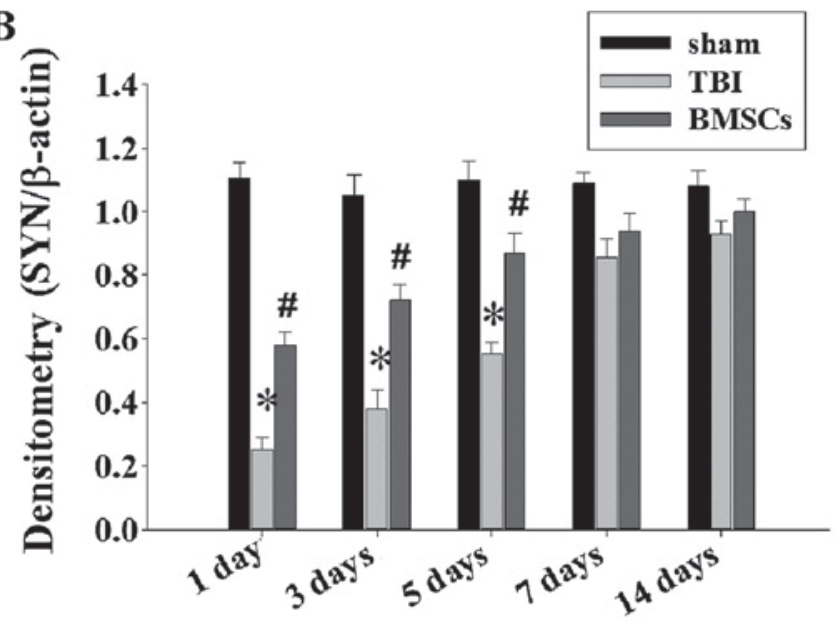

Figure 6. Effect of BMSCs on synapse protein expression. (A) Western blot images of protein expression levels of SYN in the ipsilateral cortex at 1-14 days following TBI or sham surgery. (B) Densitometry analysis of SYN bands corresponding to $\beta$-actin. Data are expressed as the mean \pm standard deviation. ${ }^{*} \mathrm{P}<0.01$ vs. sham group; ${ }^{~} \mathrm{P}<0.05$ vs. TBI group. TBI, traumatic brain injury; BMSCs, bone marrow stromal cells; SYN, synatophysin.

male rats in vivo. Following this, the expression of SRY was detected via immunofluorescence microscopy to track BMSC survival and further co-localization with NeuN and GFAP. It was demonstrated that the number of cells double-positive for SRY/NeuN or SRY/GFAP was increased in the BMSC group compared with the TBI group. This was consistent with the hypothesis that BMSCs migrate to injured areas and differentiate into neurons and astrocytes following induction of TBI in the rat.

An increase in the expression level of neurotrophic factors is considered as one of the primary underlying mechanisms to promote neuroprotection and neurorepair following damage (20). Numerous growth factors are important in brain development, including basic fibroblast growth factor, insulin-like growth factor-1, VEGF and BDNF; thus, their increased expression following brain injury may recapitulate the processes involved in brain growth and acceleration of neuronal repair. In particular, increased production of VEGF and BDNF in the injured brain has been reported to lead to functional recovery $(21,22)$. Previous studies revealed that simvastatin may significantly promote the migration of BMSCs to the injured spinal cord, increase the expression of BDNF and VEGF, reduce the lesion cavity and accelerate the recovery of hind limb function in rats (23). Song et al (24) revealed that BMSC treatment increases the expression of
SDF-1, VEGF and BDNF in the peri-infarct region following focal ischemic stroke. The present study demonstrated that BMSCs may significantly increase the expression of VEGF and BDNF around the site of injury at 14 days following TBI. Therefore, it may be hypothesized that the protective effect of BMSCs on TBI may be associated with increased expression of neurotrophic factors.

In addition, the present study demonstrated that BMSC treatment significantly increased the expression of synaptophysin at 1, 3 and 5 days compared with the TBI group. Synaptophysin has been extensively used as a marker protein to quantify the number of synapses during neuroanatomical remodeling, or following injury (25). Enhanced synaptic plasticity may be beneficial as synaptogenesis promotes neurorestorative effects and enhances functional recovery, post TBI (26). Our results demonstrated that treatment with BMSCs significantly reduced TBI-induced neuromotor impairment, as assessed by rotarod testing and mNSS. Furthermore, BMSCs increased expression of VEGF and BDNF. Protein expression levels of synaptophysin were downregulated following TBI, and this was reversed in part by treatment with BMSCs. Therefore, it may be hypothesized that BMSC treatment may contribute to the improvement of synaptic plasticity in the injured brain, enhancing motor functional outcome following TBI. In conclusion, the results of the present study revealed that treatment for TBI with BMSCs, administered by tail vein puncture, may significantly promote the migration of BMSCs to the injured brain area, reduce neuronal damage, increase the expression of BDNF, VEGF and synaptophysin, and accelerate the recovery of motor function in rats. Furthermore, these findings implicate the therapeutic potential of BMSCs as an effective treatment following TBI; however, further investigation is necessary in order to develop a novel, suitable treatment for general clinical application.

\section{References}

1. Logan TT, Villapol S and Symes AJ: TGF- $\beta$ superfamily gene expression and induction of the Runx1 transcription factor in adult neurogenic regions after brain injury. PLoS One 8: e59250, 2013.

2. Dharmasaroja P: Bone marrow-derived mesenchymal stem cells for the treatment of ischemic stroke. J Clin Neurosci 16: 12-20, 2009.

3. Nishida H, Nakayama M, Tanaka H, Kitamura M, Hatoya S, Sugiura K, Harada Y, Suzuki Y, Ide C and Inaba T: Safety of autologous bone marrow stromal cell transplantation in dogs with acute spinal cord injury. Vet Surg 41: 437-442, 2012.

4. Islam MN, Das SR, Emin MT, Wei M, Sun L, Westphalen K, Rowlands DJ, Quadri SK, Bhattacharya S and Bhattacharya J: Mitochondrial transfer from bone-marrow-derived stromal cells to pulmonary alveoli protects against acute lung injury. Nat Med 18: 759-765, 2012.

5. Zhao L, Feng Z, Hu B, Chi X and Jiao S: Ex vivo-expanded bone marrow mesenchymal stem cells facilitate recovery from chemically induced acute liver damage. Hepatogastroenterology 59: 2389-2394, 2012.

6. Li Y, Chen J, Chen XG, Wang L, Gautam SC, Xu YX, Katakowski M, Zhang LJ, Lu M, Janakiraman N and Chopp M: Human marrow stromal cell therapy for stroke in rat: Neurotrophins and functional recovery. Neurology 59: 514-523, 2002.

7. Osanai T, Kuroda S, Sugiyama T, Kawabori M, Ito M, Shichinohe $\mathrm{H}$, Kuge Y, Houkin K, Tamaki N and Iwasaki Y: Therapeutic effects of intra-arterial delivery of bone marrow stromal cells in traumatic brain injury of rats-in vivo cell tracking study by near-infrared fluorescence imaging. Neurosurgery 70: 435-444, 2012. 
8. Bae KS, Park JB, Kim HS, Kim DS, Park DJ and Kang SJ: Neuron-like differentiation of bone marrow-derived mesenchymal stem cells. Yonsei Med J 52: 401-412, 2011.

9. Tohill M, Mantovani C, Wiberg M and Terenghi G: Rat bone marrow mesenchymal stem cells express glial markers and stimulate nerve regeneration. Neurosci Lett 362: 200-203, 2004.

10. Gao X, Deng P, Xu ZC and Chen J: Moderate traumatic brain injury causes acute dendritic and synaptic degeneration in the hippocampa dentate gyrus. PLoS One 6: e24566, 2011.

11. Ye X, Yan T, Chopp M, Zacharek A, Ning R, Venkat P, Roberts C and Chen J: Combination BMSC and Niaspan treatment of stroke enhances white matter remodeling and synaptic protein expression in diabetic rats. Int J Mol Sci 14: 22221-22232, 2013

12. Aizawa-Kohama M, Endo T, Kitada M, Wakao S, Sumiyoshi A, Matsuse D, Kuroda Y, Morita T, Riera JJ, Kawashima R, et al: Transplantation of bone marrow stromal cell-derived neural precursor cells ameliorates deficits in a rat model of complete spinal cord transaction. Cell Transplant 22: 1613-1625, 2013.

13. Cui X, Chopp M, Zacharek A, Roberts C, Lu M, Savant-Bhonsale $\mathrm{S}$ and Chen J: Chemokine, vascular and therapeutic effects of combination Simvastatin and BMSCs treatment of stroke. Neurobiol Dis 36: 35-41, 2009.

14. Marmarou A, Foda MA, van den Brink W, Campbell J, Kita H and Demetriadou K: A new model of diffuse brain injury in rats. Part I: Pathophysiology and biomechanics. J Neurosurg 80: 291-300, 1994

15. Vonder Haar C, Emery MA and Hoane MR: Chronic folic acid administration confers no treatment effects in either a high or low dose following unilateral controlled cortical impact injury in the rat. Restor Neurol Neurosci 30: 291-302, 2012.

16. Gutiérrez-Fernández M, Rodríguez-Frutos B, Ramos-Cejudo J, Teresa Vallejo-Cremades M, Fuentes B, Cerdán S and Díez-Tejedor E: Effects of intravenous administration of allogenic bone marrow-and adipose tissue-derived mesenchymal stem cells on functional recovery and brain repair markers in experimental ischemic stroke. Stem Cell Res Ther 4: 11, 2013.

17. Chen J, Li Y, Wang L, Zhang Z, Lu D, Lu M and Chopp M: Therapeutic benefit of intravenous administration of bone marrow stromal cells after cerebral ischemia in rats. Stroke 32 : $1005-1011,2001$
18. Pavlichenko N, Sokolova I, Vijde S, Shvedova E, Alexandrov G, Krouglyakov P, Fedotova O, Gilerovich EG, Polyntsev DG and Otellin VA: Mesenchymal stem cells transplantation could be beneficial for treatment of experimental ischemic stroke in rats. Brain Res 1233: 203-213, 2008.

19. Bhakta S, Hong P and Koc O: The surface adhesion molecule CXCR4 stimulates mesenchymal stem cell migration to stromal cell-derived factor-1 in vitro but does not decrease apoptosis under serum deprivation. Cardiovasc Revasc Med 7: 19-24, 2006.

20. Guan J, Bennet L, Gluckman PD and Gunn AJ: Insulin-like growth factor-1 and post-ischemic brain injury. Progr Neurobiol 70: 443-462, 2003.

21. Zhang HY, Jin XB and Lue TF: Three important components in the regeneration of the cavernous nerve: Brain-derived neurotrophic factor, vascular endothelial growth factor and the JAK/STAT signaling pathway. Asian J Androl 13: 231-235, 2011.

22. Kim JH, Choi KH, Jang YJ, Kim HN, Bae SS, Choi BT and Shin HK: Electroacupuncture preconditioning reduces cerebral ischemic injury via BDNF and SDF-1 $\alpha$ in mice. BMC Complement Altern Med 13: 22, 2013.

23. Han X, Yang N, Cui Y, Xu Y, Dang G and Song C: Simvastatin mobilizes bone marrow stromal cells migrating to injured areas and promotes functional recovery after spinal cord injury in the rat. Neurosci Lett 521: 136-141, 2012.

24. Song M, Mohamad O, Gu X, Wei L and Yu SP: Restoration of intracortical and thalamocortical circuits after transplantation of bone marrow mesenchymal stem cells into the ischemic brain of mice. Cell Transplant 22: 2001-2015, 2013.

25. Brock TO and O'Callaghan JP: Quantitative changes in the synaptic vesicle proteins synapsin I and p38 and the astrocyte-specific protein glial fibrillary acidic protein are associated with chemical-induced injury to the rat central nervous system. J Neurosci 7: 931-942, 1987.

26. Pati S, Muthuraju S,HadiRA,Huat TJ,Singh S, Maletic-Savatic M, Abdullah JM and Jaafar H: Neurogenic plasticity of mesenchymal stem cell, an alluring cellular replacement for traumatic brain injury. Curr Stem Cell Res Ther 11: 149-157, 2016. 